\title{
A Novel Automatic Platform Based on Nanostructured Microfluidic Chip for Isolating and Identification of Circulating Tumor Cells
}

Hei-Jen Jou

Taiwan Adventist Hospital

\section{Li-Yun Chou}

Taiwan Adventist Hospital

Wen-Chun Chang

National Taiwan University Hospital

Hsin-Cheng Ho

National yang Ming Chiao Tung University

Wan-Ting Zhang

Cytoaurora Biotechnologies Inc.,

\section{Pei-Ying Ling}

Taiwan Adventist Hospital

Ko-Hsin Tsai

Taiwan Adventist Hospital

\section{Tze-Ho Chen}

Changhua Christian Hospital

\section{Szu-Hua Chen}

Taiwan Adventist Hospital

Pei-Hsuan Lo

Taiwan Adventist Hospital

\section{Ming Chen}

Changhua Christian Hospital

Heng-Tung Hsu ( $\nabla$ hthsu@nctu.edu.tw)

National yang Ming Chiao Tung University

\section{Research Article}

Keywords: circulating tumor cell (CTC), nanostructured microfluidic chip

Posted Date: February 23rd, 2021

DOl: https://doi.org/10.21203/rs.3.rs-226461/v1 
License: (c) (i) This work is licensed under a Creative Commons Attribution 4.0 International License. Read Full License 


\section{Abstract}

Circulating tumor cell (CTC) is commonly used as biomarker for early detection, prognostication, decision making, and evaluation of the therapeutic efficacy in cancer treatment. Unfortunately, the poor reproducibility and limited sensitivity with the CTC detection have limited its potential impact on clinical management. A reliable automated CTC detection system is therefore needed. We have designed an automated microfluidic chip-based CTC detection system consisting of three main parts: V-BioChip, Cell RevealTM enrichment and staining system, and an automatic scanning and locating system. We hypothesized this novel system can reliably detect CTC from clinical specimens. SKOV3 ovarian cancer cell line was used first to test the reliability of our system. Ten healthy volunteers, 5 patients with benign ovarian tumors, and 8 patients with epithelial ovarian cancer $(E O C)$ were recruited after obtaining written consents to validate the CTC capturing efficacy in the peripheral blood. The capture rates for spiking test in SKOV3 cells were as $48.3 \%$ and $89.6 \%$ using EpCAM antibody alone and a combined EpCAM antibody and $\mathrm{N}$-cadherin antibody, respectively. The system was sensitive to detection of low cell count and showed a linear relationship with the cell counts in our test range. The sensitivity and specificity were $62.5 \%$ and $100 \%$ when CTC was used as a biomarker for EOC. Our results demonstrated that this automatic CTC platform has a high capture rate and is feasible for detection of CTCs in EOC.

\section{Introduction}

Despite recent advances in modern medicine, we are still facing many challenges in cancer diagnosis and treatment. Tissue biopsy is the only confirmatory diagnostic method for suspicious lesions while invasive exploratory surgery is required for cancer staging. Tissue biopsy has its own limitations due to its invasiveness and the fact that some lesions are in difficult-to-reach areas. In addition, tissue biopsy cannot assess the risk of cancer metastasis, the progression of the cancer, and the response to treatment ${ }^{1}$. Furthermore, it is very difficult to detect small metastatic lesions or minimal residual diseases using even the most advanced imaging technology ${ }^{2}$. Therefore, there is an urgent need to develop more precise methods to facilitate screening, diagnosis and treatment of cancers.

CTCs are the cells shed from the primary site of the tumor and then entered the peripheral blood circulation, which has the potential to develop into new tumor foci ${ }^{3}$. Although many steps involved in cancer metastasis are still unclear, CTCs obviously play a critical role in cancer metastasis ${ }^{4,5}$. We can repeatedly take the patient's CTCs as a "real-time liquid biopsy" to predict cancer recurrence, monitor the effect of treatment, and understand potential drug resistance mechanisms ${ }^{6}$. It is estimated that there are 1-10 CTCs per $\mathrm{mL}$ of whole blood in the peripheral blood of patients with metastatic cancer ${ }^{7}$. Because of the scarcity of CTCs in the blood, it is not only technically difficult to capture CTCs from the blood of cancer patients, but also requires a lot of blood for the test.

Since 1990, there have been many technological developments and applications to enrich and identify CTCs. However, even though considerable progress has been achieved in recent years, the CTC detection technology is still not widely adopted for clinical practice because of its limitations including the scarcity 
and fragility of CTCs, the heterogeneity of CTCs, the lack of standard detection methods, and the need for sensitivity and specificity ${ }^{8}$. Most laboratories detect CTC manually which has low interrater reliability and low throughput. Therefore, the key point to overcome the current limitations is to develop an automated CTC detection technology providing unbiased result and high sensitivity will benefit clinicians taking care of the cancer patients. The rapidly improving microfluidic-based technology over the last ten years can be a sensitive, efficient, and fully automated system with a portable size $e^{8,9}$.

Ovarian cancer is a common cancer in women with extremely poor prognosis. With the current diagnostic techniques, more than half of ovarian cancers are diagnosed at advanced stage ${ }^{10}$. Therefore, there is an urgent need for new diagnostic tools to assist in the management of ovarian cancer, including: early diagnosis, evaluation before and after surgery, prognostic judgment, detection of minimal residual disease or early recurrence, assessment of treatment response, and even drug selection. The purpose of this study is to evaluate the applicability of a newly designed, fully automated microfluidic CTC platform, in the clinical evaluation of ovarian cancer.

In the present study, we used an automatic microfluidic immunoaffinity-based system to achieve reliable reproducibility with high throughput. This automated system consists of several parts, including a nanostructured microfluidic biochip, an automatic CTC enrichment and staining system, and an automatic CTC scanning and locating system. The capture efficiency of the automatic system was tested by spiking tests of SKOV3 ovarian cancer cell line. A preliminary test for clinical feasibility on epithelial ovarian cancer (EOC) from consented human volunteers was also conducted.

\section{Results}

The enrichment and staining processes were achieved within as short as 4 hours in the Cell Reveal ${ }^{\mathrm{TM}}$ system but overnight handing will not change the readouts. The automatic on-chip scanning and cell locating by CAT took about 15 minutes.

V-BioChip has excellent capture ability for target cells. Figure 1a demonstrates the gross appearance of a V-BioChip and the image of an SKOV3 cell captured by V-BioChip under a scanning electron microscope (SEM) at 5,000X magnification (Fig 1b). It can be seen from the SEM image that there were many microvilli on the surface of the SKOV3 cell, and some microvilli firmly adhered to the nanopillars of VBioChip. The captured SKOV3 cells had intact morphology without distortion, which means that VBioChip can capture the targeted cells under the automatic process without obvious damage.

Because of the scarcity of CTC, capture efficiency is critical for a successful CTC test platform. With EpCAM antibody alone as the capture antibody, the recovery rate was $48.3 \%$ with a SD of $11.7 \%$.

Considering the mesenchymal characteristics of SKOV3 cells, we used a mixture of an epithelial marker antibody (anti-EpCAM antibody) as well as an EMT marker antibody (anti-N-cadherin antibody) to make a capture antibody cocktail (Combi). The Combi strategy markedly increased the capture rate to $89.6 \%$ (SD: 11.8\%) (Fig 2a). 
The analysis of the linear relationship between the number of spiking cells and the number of captured cells at low spiking cell numbers is also an important indicator of the capture efficiency of the CTC platform. The present study showed a high recovery rate and a linear relationship even in low spiking cell numbers $(39-625)$. The linear regression was $y=0.7888 x \pm 1.5009(R 2=0.9639)$ with an average recovery rates $73.5 \%$ (SD: $11.8 \%$ ) (Fig 2 b).

The results of clinical validity tests are summarized in Table 1 and Fig 3. No CTCs were detected in the peripheral blood of the ten healthy subjects. As for patients with benign ovarian tumors and EOC, the

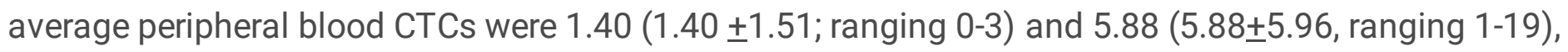
respectively (Fig 3a). If a cutoff of $\geq 4$ CTCs in $4 \mathrm{ml}$ of blood is used to screen for EOC, the sensitivity is $62.5 \%$ and the specificity is $100 \%$, respectively.

Our results revealed that neither healthy subjects nor patients with benign ovarian tumors could detect CD13+ CTCs in the peripheral blood. The average number of CD13+ CTCs in the peripheral blood of EOC patients was $3.375 / 4 \mathrm{ml}$ (ranging: $0-19$ ) (Fig $3 b$ ). The results showed a sensitivity of $62.5 \%$ and a specificity of $100 \%$, respectively, if we used the presence of CD13+ CTCs or not as the cutoff. Further analysis showed a sensitivity of $75 \%$ and a specificity of $100 \%$, respectively, if we used the cutoff as $\geq 4$ CTCs or the presence of CD13+ CTCs in $4 \mathrm{ml}$ of blood.

\section{Discussion}

In the present study, we show a fully automated CTC detection platform that can efficiently capture, identify, scan, locate, enumerate and even characterize CTCs on-chip. The development of fully automated systems has greatly increased the possibility of CTC testing being utilized in clinical practice. This study also preliminarily tested the validity of the platform for patients with EOC, and found that its considerable potential in the detection of CTCs in EOC patients and could assist the treatment decision in EOC and even other cancers.

Due to the rarity of CTCs in the peripheral blood of cancer patients, high enrichment efficiency is critical for CTC detection platform. The design and manufacture of microfluidic chips plays an important role in the entire system. V-BioChip is an immunoaffinity-based microfluidic chip which mainly based on the affinity binding between CTCs and the surface of the nanopillar on the chip. The surface of nanopillars is coated with biotinylated PLL-g-PEG + streptavidin and the target cells are pre-labeled with biotinylated monoclonal antibody. Nanotexturing improved the adhesion of microvilli and invadopodia of CTCs to the surface of nanopillars which was functionalized with streptavidin ${ }^{11}$.

Epithelial cell adhesion molecule (EpCAM) is a cancer-related antigen. Cancer tissues of epithelial origin often overexpress EpCAM ${ }^{12-14}$. Therefore, in the past few decades, EpCAM has been used by many enrichment technologies as the main cell membrane marker to isolate CTCs, and considerable progress has been made in the use of EpCAM-based CTC detection technologies. However, there are many reasons why EpCAM-based technologies cannot effectively detect CTCs. The expression of EpCAM in variant 
cancers is quite different ${ }^{15}$. In addition, epithelial-to-mesenchymal transition (EMT) is a key step of the metastatic cascade. This process allows epithelial cancer cells to acquire some mesenchymal characteristics while losing epithelial cell phenotype, resulting in a decrease in the cell's EpCAM expression ${ }^{16,17}$. For example, the expression of EpCAM in patients with ovarian cancer depends on their histological subtypes. Overall, 73\% of ovarian cancers overexpress EpCAM, but serous ovarian cancers have a lower EpCAM overexpression rate $(55 \%)^{15}$.

Due to the downregulation of CTC EPCAM expression during the EMT process, the use of EpCAM-based enrichment techniques may not efficiently capture the CTC populations that are currently EMT ${ }^{18}$. SKOV3 is an EOC cell line with mesenchymal phenotype. It is reasonable that the present study shows a low capture efficiency of $48 \%$ for SKOV3 cells when using a single epithelial antibody as the capture antibody. For cancer cells undergoing EMT, further strategies must be taken to achieve better capture efficiency.

The combined use of epidermal and mesenchymal markers not only increases the probability of capturing CTCs of different phenotypes, but also reduces the omission of EpCAM-negative CTCs. Po and his colleagues demonstrated that adding anti-N-cadherin antibodies to anti-EpCAM antibodies can increase the efficiency of capturing CTCs when using immunomagnetic beads as a strategy for isolating $\mathrm{CTCs}^{19}$. Another study showed that using five different types of antibodies on the CTC platform can offer a significant improvement in cell-isolation efficiency, even from tiny amounts of blood ( $250 \mu \mathrm{L}-1 \mathrm{~mL})$ or in non-metastatic breast cancer ${ }^{20}$. The present research also reached similar conclusion as the previous studies. Using the Combi strategy by mixing an epithelial antibody and a mesenchymal antibody as the enrichment antibody combination can significantly overcome the adverse effect of downregulation of EpCAM expression caused by EMT on the CTC capture efficiency of the microfluidic system and achieve an optimal capture rate. Most studies were conducted by using PBMCs suspensions of cancer cells that mimic actual conditions. The donor's blood can interact with the spiked cells and markedly affect the recovery efficiency. Our results show that the automated CTC platform combined with V-BioChip and Cell Reveal ${ }^{\mathrm{TM}}$ system can achieve excellent CTC capture efficiency of $90 \%$ by using Combi strategy. Even in low cell count, the system also demonstrated excellent capture efficiency and device linearity with $\mathrm{R}^{2}$ of $96 \%$.

Current methods for CTCs identification and analysis depend on immunofluorescence technologies and fluorescence microscopic imaging by characterizing the tumor cells with specific markers. Up until now, most of the microfluidic chip-based CTCs platforms still rely on manual operations for cell screening and interpretation after immunofluorescent staining of target cells, which is time-consuming and causes inconsistent results. So far, only a limited number of automated CTCs microscope systems have been reported $^{21}$. The automated CTCs scanning and identification system can complete the scanning of the target cells in a short time, which not only saves time and manpower, but also helps to establish standard processes. Our state-of-the-art automated microscope control system and CTCs scanning and identification system can automatically number and locate target cells in addition to automatic scanning and identification of target cells. Numbering and positioning functions can find specific target cells when 
manual inspection or re-evaluation is required. In addition, these functions can re-locate specific cells on the chip in a very short period of time, which is very useful when single cell analysis is required.

In the face of the extremely poor prognosis of ovarian cancer, CTC has the potential to provide more information beyond the current testing technology to assist treatment decisions. In the past decade, there have been many studies showing the use of CTCs as a "real-time liquid biopsy" for the diagnosis and treatment of ovarian cancer. However, these studies show a low positive rate of CTCs and a low median of $\mathrm{CTCs}^{8,22-24}$. The possible reason is the early onset of peritoneal dissemination in EOC, with only one third of the patient has distant metastases, leading to insufficient CTCs in the peripheral blood to be detected $^{25,26}$. In addition, most of these studies do not report the sensitivity and specificity of the technology used ${ }^{19,27-29}$. Therefore, the clinical application of CTCs in management of EOC still needs further confirmation.

In addition to enumeration of CTCs, single-cell phenotypic characterization can provide additional biochemical information to assist clinical diagnosis and treatment decisions ${ }^{30}$. Aminopeptidase $\mathrm{N}$ (CD13) is a surface marker of cancer stem cells (CSC). It plays a role in cancer cell invasion and is also a candidate for treatment resistance, recurrence and poor prognosis, which is highly expressed in early stage of $\mathrm{EOC}^{31,32}$. Van Hensbergen and his colleagues found that the expression of CD13 in EOC was associated with the histological subtype: CD13 expression in tumor cells was observed in $80 \%$ of the patients with a serous carcinoma and $100 \%$ of the patients with a mucinous carcinoma and in only $20 \%$ of the clear cell carcinoma patients ${ }^{32,33}$. To the best of our knowledge, this is the first attempt to isolate and identify CD13+ CTCs in EOC patients.

Although the case number in the present study is limited, there are several meaningful findings. First, no matter which cutoff is used, there is a high sensitivity, which can greatly simplify the interpretation of clinicians. Second, the positive rate of CD13+ CTCs in serous adenocarcinoma is consistent with the histological study by Van Hensbergen. Third, CTCs or CD13+ CTCs can be detected even in the early stage (stage I) EOC. It means that CTCs have appeared in the blood at least in some early EOCs, indicating the possibility of micrometastasis even in the early state of EOC by surgical staging. Finally, due to the full automation of the laboratory processes, it is technically possible to simultaneously detect one or more additional cell markers in. In other words, it has the potential to provide a comprehensive cell profiling for clinical applications, but more experimental validation is still needed.

In conclusion, as the advantage of microfluidic chips and automated systems, CTCs detection has become more and more efficient and accurate. Although many microfluidic-based methods have been developed in the past decade, most of them cannot be applied clinically due to lack of automation, low throughput, and high cost of microfluidic chips. This study shows that the automated Cell Reveal ${ }^{\mathrm{TM}}$ system has the potential for widespread clinical application to aid in the treatment decisions of EOC and even other cancers. However, in spite of the very promising preliminary results, more clinical experience is needed to reach further conclusions due to the complexity and heterogeneity of cancers. 


\section{Methods}

\section{Cell line preparation}

A human ovarian adenocarcinoma cell line, SKOV3 (ATCC® HTB-77 ${ }^{\mathrm{TM}}$, Manassas, VA, US), was used for the cell spiking test. The SKOV3 cells were cultured in McCoy's 5A medium (BioConcept, Allschwil, Switzerland), supplemented with $10 \%$ fetal bovine serum (FBS), 100 units/mL penicillin (Gibco, Grand Island, NY, USA). Peripheral blood mononuclear cells (PBMCs) were separated from the whole blood of healthy volunteers by a density gradient centrifugation method.

Two different enrichment strategies were used for spiking test, including biotinylated anti-EpCAM antibody only, and an antibody cocktail combined with biotinylated anti-EpCAM antibody and biotinylated anti-N-cadherin antibody (Combi). Prior to their mixture, both SKOV3 cells and PBMCs were incubated with anti-EpCAM antibody or Combi cocktail at $370 \mathrm{C}$ for 45 minutes in a $15 \mathrm{~mL}$ centrifuge tube. Then $3 \mathrm{~mL}$ DPBS was added to each tube and the tubes were centrifuged at $300 \mathrm{~g}$ for 10 minutes to collect the cell pallets and remove free antibodies. The cell mixture for spiking test was prepared by spiking $5 \star 10^{3}$ SKOV3 cells into PBMCs of $2 \mathrm{~mL}$ whole blood origin in $200 \mu \mathrm{L}$ of DPBS. The SKOV3 cell number was counted using Countess ${ }^{\mathrm{TM}}$ II FL Automated Cell Counter (ThermoFisher, Waltham, MA, USA).

\section{Microfluidic chip}

The V-BioChip (CytoAurora Inc., HsinChu, Taiwan) is a silicon-based chip with nano-pillar arrays on the inside of microfluidic chambers (Fig 1). The structure and production process of the predecessor of $\mathrm{V}$ BioChip, Cral Chip, has been described in detail in Ma's report ${ }^{11}$. To improve the chip's capture efficiency, we modified original design of Coral Chip to adjust the distance between the nano-pillars on the microfluidic chip and the shape of the nano-pillars. In brief, metal-assisted chemical etching (MACE) technology was used to form matrix-arranged nano-pillars on the chip surface. The tip of the pillars is modified into a shape of volcanic cone to increase the contact surface between the target cells and the nano-pillars, in attempt to reduce cell damage. Thereafter, the polyethylene glycol-biotin (PEG-biotin) layer was modified on the surface of the wafer by vapor deposition method. Streptavidin, a tetrameric protein with high binding affinity to biotin ${ }^{34}$, was then attached to the biotin end of the PEG-biotin using liquid deposition technology. The streptavidin-biotin interaction is one of the strongest non-covalent biological interactions currently known and can markedly increase the capture efficiency to the target cells $s^{35,36}$. When the mixed cell suspension flows over the chip, the target cells will be captured by the V-BioChip, and most other cells will be washed away.

\section{Cell spiking test}

The Cell Reveal ${ }^{\mathrm{TM}}$ machine (CytoAurora Inc., HsinChu, Taiwan) was used for the enrichment and staining of the CTCs. Before the test, the V-Biochip was set up inside the machine. The mixed cell suspension of SKOV3 and PBMCs was injected into the Cell Reveal ${ }^{\mathrm{TM}}$ system, and the system automatically processed the CTC enrichment and staining procedures. The inputted cell mixture was then fixed in $4 \%$ 
paraformaldehyde. Subsequently, $0.1 \%$ of Triton X-100 (ThermoFisher, Waltham, MA, USA) and $2 \%$ BSA (Bovine serum albumin) were added to increase the cellular permeability. The cell mixture passes through the V-BioChip at a flow rate of $0.6 \mathrm{~mL} / \mathrm{h}$, allowing the target cells to fully contact the chip to achieve an optimal capture rate. As the cell-rich centrate flows through the microfluidic chips, the streptavidin on the chips captures the target cells bound with biotinylated anti-EPCAM antibodies. The process of CTC enrichment and staining were done overnight to achieve the best staining. But this process can be completed within four hours.

In order to distinguish CTCs from white blood cells, microfluidic chips were incubated with anti-EpCAM antibody (R\&D Systems, Minneapolis, MN, USA) conjugated with FITC (for the detection of CTCs), as well as anti-CD45 (Agilent, Santa Clara, CA, USA) antibody conjugated with TRITC (for the detection of white blood cells) and 4',6-diamidino-2-phenylindole (DAPI; Invitrogen, Carlsbad, CA, USA) (for the detection of nucleated cells). The CTC was defined as an EpCAM+/CD45-/DAPI+ intact cell.

After the enrichment and staining steps, V-BioChip is sent to the CTC scanning platform for further analysis. This state-of-the-art CTC scanning platform is composed three main parts, including a modified upright fluorescent microscope and two sets of self-developed software (CytoAcqlmages system for automatic image scanning and CAT automatic cell identification system). The upright fluorescent microscope (Leica DM6 B, Leica Microsystems GmBH, Wetzlar, Germany) is equipped with Leica HC PL APO 10x / 0.45 microscope objective, spectra III light engine (Lumencor, Beaverton, OR, USA) with wavelengh range: $380 \mathrm{~nm} 750 \mathrm{~nm}$, Andor Zyla 4.2 sCMOS camera and Marzhauser scanning stage for 4 slides and is controlled by the CytoAcqlmages (CAl) system. The CAl system can be used with any brand of microscope, light controller, motorized XYZ stages and SCMOS camera.

Under the control of CAl system, the microscope automatically focuses and scans the V-BioChip, as well as activates the exposure with a fluorescent carousel (Fig 4a). A fully automatic segmented photographing method was adopted to obtain high-resolution full-area images of V-BioChips. Then, the image files were montaged together to form a whole biochip TIFF image file.

V-BioChip is pre-installed with special positioning marks. When the CAl system focuses on these marks, it also records the coordinate information to the full chip image file. When the image interpretation system reads the chip image file, it can use the coordinate information to calculate the specific position of the specific cell on the chip. In addition, this precise positioning function can also help to find specific cells on the chip again, which can assist in re-evaluating specific cells and even further single-cell analysis.

The full chip image files were then transmitted to CAT system (Fig 4b). CAT system can identify target cells according to the immunofluorescence staining on the cells by using pre-set parameters and deep learning Al function. Count-in/filter-out criteria were used to identify the cells while EpCAM+/CD45-/DAPI+ for CTCs and EpCAM-/CD45+/DAPI+ for WBCs (Fig 5a).

Three separate spiking tests were performed for both the single ant-EpCAM antibody and Combi strategy. The results are expressed as the mean recovery rate \pm standard deviation (SD). 


\section{Linearity between the numbers of captured cells and spiked cells}

The relationship between the captured cell numbers and the CTC numbers was analyzed by spiking SKOV3 cells into DPBS was analyzed. We incubated 625 SKOV3 cells with $5 \mu \mathrm{L}$ cocktail capture antibodies in $200 \mu \mathrm{L}$ DPBS at $37^{\circ} \mathrm{C}$ for $45 \mathrm{~min}$ and then centrifuged with $400 \mathrm{~g}$ for $10 \mathrm{~min}$. A serial of 2 -fold dilutions was performed to allow for linear regression analysis of the estimated cell numbers being 625 , $312,156,78$ and 39 . The diluted cell suspensions were then injected into Cell Reveal ${ }^{\mathrm{TM}}$ System to capture SKOV3 cells by streptavidin-coated chip with a flow speed of $0.6 \mu \mathrm{L} / \mathrm{min}$. The captured cells were stained by EpCAM/CD45/DAPI on chip and subsequently were scanned and counted by the automatic CAT system.

Each spiking cell number is tested twice. In addition to linear regression analysis, the recovery rate of each test was also calculated. The results of recovery rate were expressed as mean recovery rate \pm SD.

\section{Feasibility study}

In order to examine the clinical feasibility of the test, we recruited participants to join the study, including 10 healthy subjects without any type of ovarian tumor, 5 women with benign ovarian tumor and 8 patients with epithelial ovarian cancer (EOC). FIGO criteria were used for the staging of EOC. The study has been approved by the IRB committee in the Taiwan Adventist Hospital and Changhua Christian Hospital. All methods were carried out in accordance with relevant guidelines and regulations and each participant completed a written consent before they received the test.

In this study, anti-CD13 antibody (Abcam, Cambridge, UK) was also used for ICC staining to identify CTCs that carry CD13 (Aminopeptidase N) surface marker. CD13 is a function marker for cancer stem cell. When an intact cell shows ICC staining of CD13+/EpCAM+/CD45-/DAPI+, it is defined as a CD13+CTC (Fig 5b).

\section{Declarations}

Additional information

Competing Interests Statement:

Ming Chen is the Honorary Cofounder of CytoAurora Biotechnologies Inc., Hsinchu

Science Park, Hsinchu, Taiwan. Hsin-Cheng Ho and Wan-Ting Zhang are employees of CytoAurora Biotechnologies Inc., Hsinchu Science Park, Hsinchu, Taiwan. The experimental equipment is provided by CytoAurora Biotechnologies Inc., Hsinchu Science Park, Hsinchu, Taiwan. All other authors declare no conflict of interests.

\section{References}


1. Quandt, D. et al. Implementing liquid biopsies into clinical decision making for cancer immunotherapy. Oncotarget 8, 48507-48520 (2017).

2. Pantel, K. \& Alix-Panabières, C. Liquid biopsy and minimal residual disease-latest advances and implications for cure. Rev. Clin. Oncol. 16, 409-424 (2019).

3. Yap, T. A., Lorente, D., Omlin, A., Olmos, D. \& De Bono, J. S. Circulating Tumor Cells: A Multifunctional Biomarker. Cancer Res. 20, 2553-2568 (2014).

4. Valastyan, S. \& Weinberg, R. A. Tumor metastasis: molecular insights and evolving paradigms. Cell 147, 275-292 (2011).

5. Lambert, A. W., Pattabiraman, D. R. \& Weinberg, R. A. Emerging biological principles of metastasis. Cell 168, 670-691 (2017).

6. Woo, D. \& Yu, M. Circulating tumor cells as "liquid biopsies" to understand cancer metastasis. Res. 201, 128-135 (2018).

7. Zieglschmid, V., Hollmann, C. \& Böcher, O. Detection of disseminated tumor cells in peripheral blood. Rev. Clin. Lab. Sci. 42, 155-196 (2005).

8. Zou, D. \& Cui, D. Advances in isolation and detection of circulating tumor cells based on microfluidics. Cancer Biol. Med. 15, 335-353 (2018).

9. Nagrath, S. et al. Isolation of rare circulating tumour cells in cancer patients by microchip technology. Nature 450, 1235-1239 (2007).

10. Siegel, R. L., Miller, K. D. \& Jemal, A. Cancer statistics, 2020. CA Cancer J. Clin. 70, 7-30 (2020).

11. Ma, G. -C. et al. A silicon-based coral-like nanostructured microfluidics to isolate rare cells in human circulation: validation by SK-BR-3 cancer cell line and its utility in circulating fetal nucleated red blood cells. Micromachines 10, 132 (2019).

12. Schmelzer, E. \& Reid, L. M. EpCAM expression in normal, non-pathological tissues. Biosci. 13, 30963100 (2008).

13. Went, P. T. et al. Frequent EpCam protein expression in human carcinomas. Pathol. 35, 122-128 (2004).

14. Osta, W. A. et al. EpCAM is overexpressed in breast cancer and is a potential target for breast cancer gene therapy. Cancer Res. 64, 5818-5824 (2004).

15. Spizzo, G. et al. EpCAM expression in primary tumour tissues and metastases: an immunohistochemical analysis. Clin. Pathol. 64, 415-420 (2011).

16. Jie, X. -X., Zhang, X. -Y. \& Xu, C. -J. Epithelial-to-mesenchymal transition, circulating tumor cells and cancer metastasis: Mechanisms and clinical applications. Oncotarget 8, 81558 (2017).

17. Mohtar, M. A., Syafruddin, S. E., Nasir, S. N. \& Yew, L. T. Revisiting the Roles of Pro-Metastatic EpCAM in Cancer. Biomolecules 10, 255 (2020).

18. Gorges, T. M. et al. Circulating tumour cells escape from EpCAM-based detection due to epithelial-tomesenchymal transition. BMC cancer 12, 178 (2012). 
19. Po, J. W. et al. Improved ovarian cancer EMT-CTC isolation by immunomagnetic targeting of epithelial EpCAM and mesenchymal N-cadherin. Circ. Biomark. 7, 1849454418782617 (2018).

20. Hong, W., Lee, S., Chang, H. J., Lee, E. S. \& Cho, Y. Multifunctional magnetic nanowires: a novel breakthrough for ultrasensitive detection and isolation of rare cancer cells from non-metastatic early breast cancer patients using small volumes of blood. Biomaterials 106, 78-86 (2016).

21. Aguilar-Avelar, C. et al. High-throughput automated microscopy of circulating tumor cells. Rep. 9, 1-9 (2019).

22. Liu, J. F. et al. Predictive value of circulating tumor cells (CTCs) in newly-diagnosed and recurrent ovarian cancer patients. Oncol. 131, 352-356 (2013).

23. Suh, D. H. et al. Circulating tumor cells in the differential diagnosis of adnexal masses. Oncotarget $\mathbf{8}$, 77195 (2017).

24. Obermayr, E. et al. Circulating tumor cells: potential markers of minimal residual disease in ovarian cancer? a study of the OVCAD consortium. Oncotarget 8, 106415 (2017).

25. Gao, Q. et al. Heterotypic CAF-tumor spheroids promote early peritoneal metastasis of ovarian cancer. Exp. Med. 216, 688-703 (2019).

26. Van Berckelaer, C. et al. Current and future role of circulating tumor cells in patients with epithelial ovarian cancer. J. Surg. Oncol. 42, 1772-1779 (2016).

27. Zhang, X. et al. Analysis of circulating tumor cells in ovarian cancer and their clinical value as a biomarker. Cell Physiol. Biochem. 48, 1983-1994 (2018).

28. Lee, M. et al. Predictive value of circulating tumor cells (CTCs) captured by microfluidic device in patients with epithelial ovarian cancer. Oncol. 145, 361-365 (2017).

29. Rao, Q. et al. Detection of circulating tumour cells in patients with epithelial ovarian cancer by a microfluidic system. J. Clin. Exp. Pathol. 10, 9599-9606 (2017).

30. Gonzalez, V. D. et al. Commonly occurring cell subsets in high-grade serous ovarian tumors identified by single-cell mass cytometry. Cell Rep. 22, 1875-1888 (2018).

31. Surowiak, P. et al. Expression of aminopeptidase N/CD13 in human ovarian cancers. J. Gynecol. Cancer 16, 1783-1788 (2006).

32. Wickström, M., Larsson, R., Nygren, P. \& Gullbo, J. Aminopeptidase N (CD13) as a target for cancer chemotherapy. Cancer Sci. 102, 501-508 (2011).

33. Van Hensbergen, Y. et al. Reduced growth, increased vascular area, and reduced response to cisplatin in CD13-overexpressing human ovarian cancer xenografts. Clin. Cancer Res. 10, 1180-1191 (2004).

34. Lakshmipriya, T., Gopinath, S. C. \& Tang, T. H. Biotin-Streptavidin Competition Mediates Sensitive Detection of Biomolecules in Enzyme Linked Immunosorbent Assay. PLoS One 11, e0151153 (2016).

35. Dundas, C.M., Demonte, D. \& Park, S. Streptavidin-biotin technology: improvements and innovations in chemical and biological applications. Appl. Microbiol. Biotechnol. 97, 9343-9353 (2013).

36. Stayton, P. S. et al. Streptavidin-biotin binding energetics. Eng. 16, 39-44 (1999). 


\section{Table}

Table 1. Summary of CTC and CD13+ CTC counts in patients with benign ovarian tumor or epithelial ovarian cancer

\begin{tabular}{|c|c|c|c|c|c|}
\hline Case No & $\overline{\text { Age }}$ & Diagnosis & Staging & $\begin{array}{l}\text { No of } \\
\text { CTCs }\end{array}$ & $\begin{array}{l}\text { No of CD13+ } \\
\text { CTCs }\end{array}$ \\
\hline \multicolumn{6}{|l|}{ Benign } \\
\hline 1 & 45 & Endometrioma & - & 3 & 0 \\
\hline 2 & 48 & Endometrioma & - & 1 & 0 \\
\hline 3 & 42 & Mature teratoma & - & 0 & 0 \\
\hline 4 & 32 & Endometrioma & - & 3 & 0 \\
\hline 5 & 34 & Endometrioma & - & 0 & 0 \\
\hline \multicolumn{6}{|l|}{ Malignant } \\
\hline 1 & 56 & $\begin{array}{l}\text { High grade serous } \\
\text { cystadenocarcinoma }\end{array}$ & I & 6 & 6 \\
\hline 2 & 56 & $\begin{array}{l}\text { High grade serous } \\
\text { cystadenocarcinoma }\end{array}$ & $\mathrm{I}$ & 1 & 1 \\
\hline 3 & 21 & Endometrioid carcinoma & $\mathrm{I}$ & 5 & 0 \\
\hline 4 & 61 & Endometrioid carcinoma & $\mathrm{I}$ & 19 & 8 \\
\hline 5 & 67 & $\begin{array}{l}\text { High grade serous } \\
\text { cystadenocarcinoma }\end{array}$ & II & 2 & 0 \\
\hline 6 & 60 & Clear cell carcinoma & III & 1 & 0 \\
\hline 7 & 48 & $\begin{array}{l}\text { High grade serous } \\
\text { cystadenocarcinoma }\end{array}$ & III & 4 & 3 \\
\hline 8 & 58 & Endometrioid carcinoma & III & 9 & 9 \\
\hline
\end{tabular}

Figures 

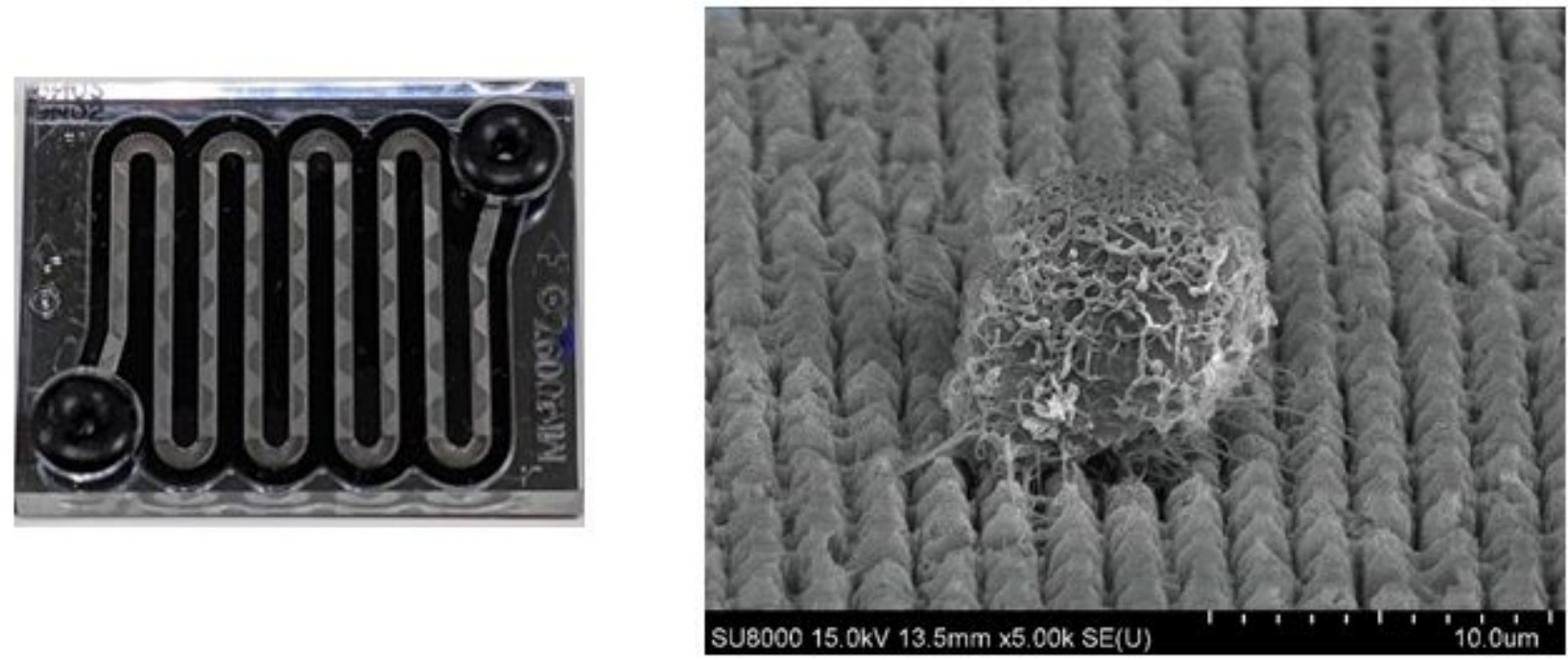

\section{Figure 1}

The gross appearance of V-BioChip and the scanning electron micrograph (SEM) of SKOB3 cell on the chip. (a). The silicon-based microfluidic V-BioChip. The microfluidic channel on the chip winded into nine segments to increase the length of the channel, so that the blood sample can fully contactact with the nanopillars on the microfluidic channel. (b) The SEM photo of an intact ovarian cancer cell (SKOV3 cell) on the V-BioChip. It can be seen that the microvilli (white arrows) of SKOV3 cells are firmly attached to the surface of the nano-pillars of the V-BioChip. Note that the top of the nano-pillars is modified to the shape of the volcanic cone to increase the contact area with the target cell to increase the capture efficiency.

\section{Capture rate on SKOV3 cells lines}

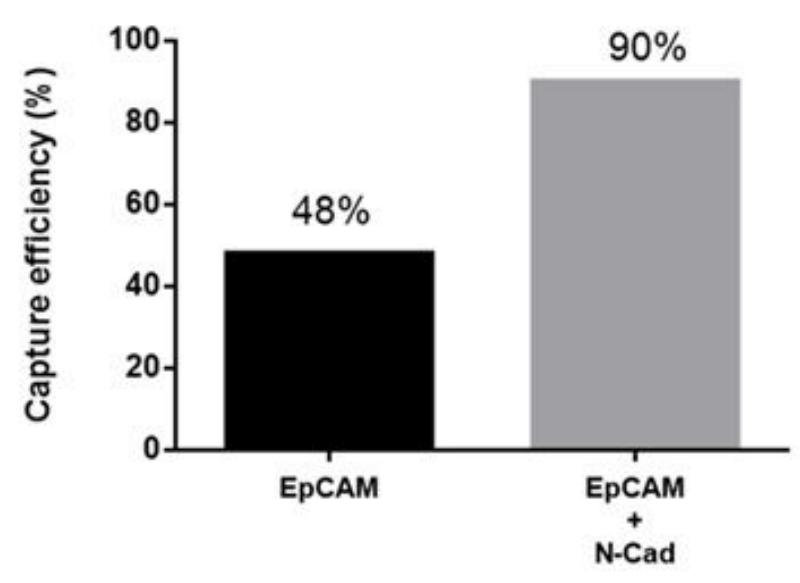

Capture Antibody

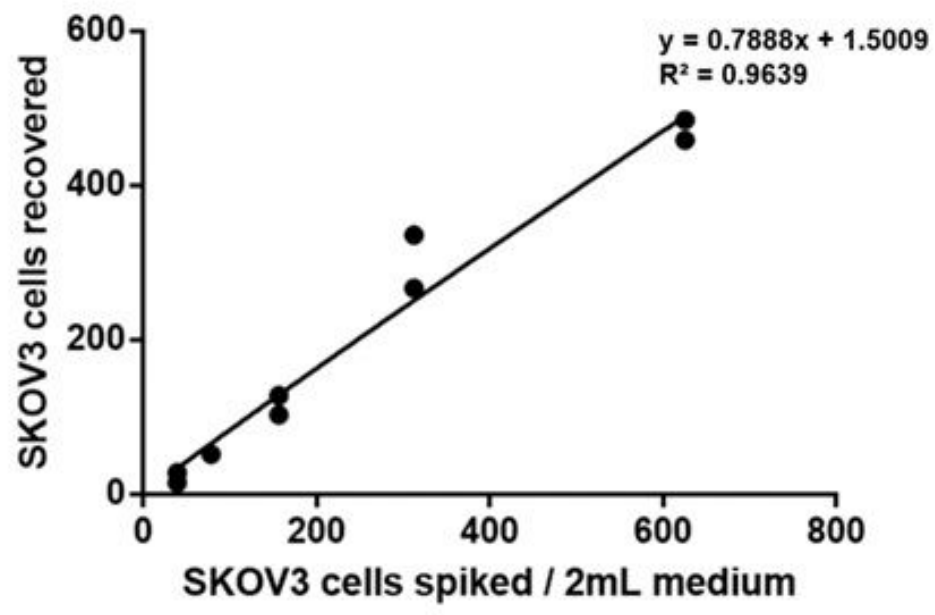

Figure 2 
Capture efficiency of SKOV3 cells. (a). Capture efficiency of SKOV3 cells by using a single anti-EpCAM antibody or a combination of anti-EpCAM antibody and anti-N-cadherin antibody as capture strategy. The bar reveals the overall efficiency, defined as the target cell number counted by the automatic CAT system as a proportion of the spike cell number, in both PBMCs. CTC is defined as an intact

EpCAM+/CT45-/DAPI+ cells. (b) The linearity of Cell RevealTM system in low spiking number by spiking SKOV3 cells in media. Linear regression was calculated between the number of the captured cells ( $Y$-axis) and the number of spiked cells (X-axis).
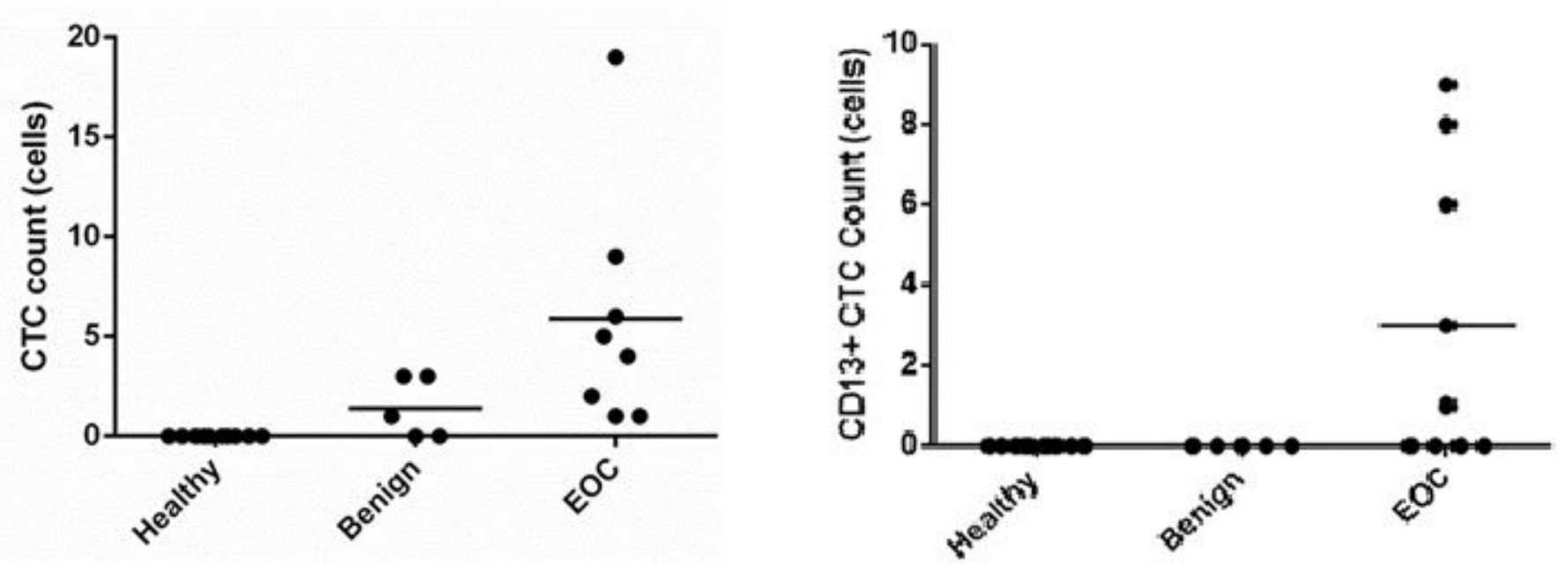

Figure 3

CTC counts and CD13+ CTC counts in healthy volunteers, patients of benign ovarian tumor and EOC patients. (a) No CTCs were detected in the peripheral blood of the ten healthy subjects. As for patients with benign ovarian tumors and EOC, the average peripheral blood CTCs were $1.40(1.40+1.51$; ranging 0 3 ) and 5.88 (5.88+5.96, ranging 1-19), respectively. (b) Neither healthy volunteers nor patients with benign ovarian tumors could detect CD13+ CTCs in the peripheral blood. The average number of CD13+ CTCs in the peripheral blood of EOC patients was $3.375 / 4 \mathrm{ml}$ (ranging: 0-19) 

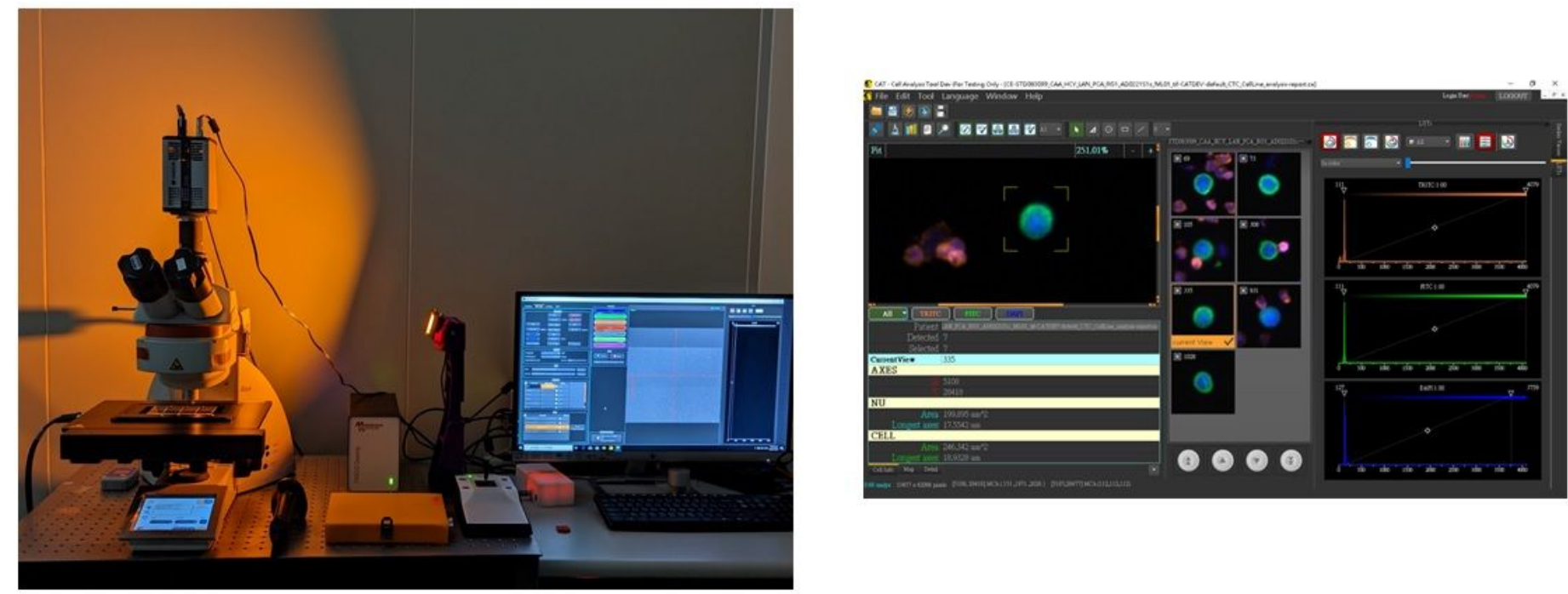

Figure 4

The automatic scanning, locating and cell analysis system (a) A Leica DM6 B upright microscope equipped with Leica HC PL APO 10x /0.45 microscope objective, spectra III light engine with wavelenght: 380nm 750nm, Andor Zyla 4.2 sCMOS camera and Marzhauser scanning stage for 4 slides. This microscope is controlled by the CytoAcqImages system to perform automatic scanning of V-BioChip and positioning of target cells; (b) The CAT (Cell Analysis Tools) system, which can identify target cells according to the immunofluorescence staining on the cells by using pre-set parameters and deep learning Al function.
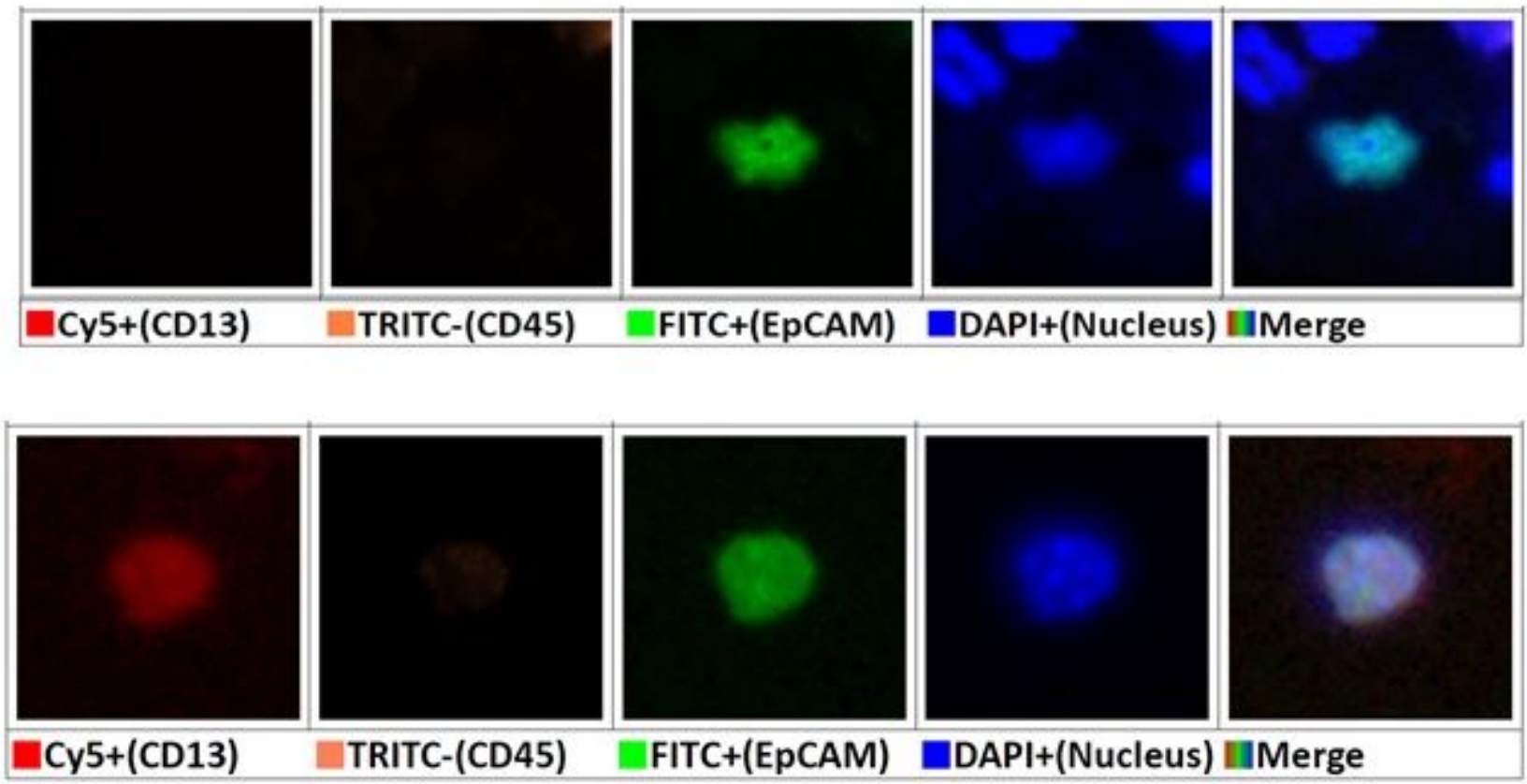

Figure 5 
Immunofluorescence staining of circulating tumor cells and characterization with cancer stem cell specific molecular marker CD13 (a) CTC from a patient with endometrioma stained CD13-/EpCAM+/CD45-/DAPI+; (b) CTC from an EOC patient (high-grade serous cystadenocarcinoma, FIGO stage Ic3) showed CD13+/EpCAM+/CD45-/DAPI+ staining. 